\title{
Degree of polarization in quantum optics
}

\author{
Alfredo Luis* \\ Departamento de Óptica, Facultad de Ciencias Físicas, Universidad Complutense, 28040 Madrid, Spain
}

(Received 14 December 2001; published 15 July 2002)

\begin{abstract}
Quantum optics entails polarization properties that cannot be fully described by the classical Stokes parameters. In this work, we characterize the polarization of classical as well as quantum fields by means of a probability distribution on the Poincare sphere. This serves to define the degree of polarization of a field state as the distance between the corresponding polarization distribution and the uniform distribution representing unpolarized light. We apply this definition to relevant quantum field states such as SU(2) coherent, squeezed, number, and phase states.
\end{abstract}

DOI: 10.1103/PhysRevA.66.013806

PACS number(s): 42.50.Dv, 03.65.Ca, 42.25.Ja

\section{INTRODUCTION}

The Stokes parameters provide a convenient description of the polarization of light in classical optics. In particular, they allow us to classify the states of light according to a degree of polarization [1]. This formalism can be extended to the quantum domain, where the Stokes parameters become the mean values of the Stokes operators.

The Stokes parameters are proportional to the secondorder correlations of the field amplitudes. While this may be enough for most classical problems, for quantum fields, higher-order correlations are crucial. Because of this, the Stokes parameters do not distinguish between very different quantum states having remarkably dissimilar polarization properties [2-6]. For example, this is the case of polarization squeezing, which is actually defined by the fluctuations of the Stokes operators around their mean values [7-10]. Moreover, the classical degree of polarization can be zero for field states that cannot be regarded as unpolarized [2-5].

In this work, we study a full characterization of polarization by means of a probability distribution defined on the surface of the Poincare sphere. As a matter of fact, the existence of such a probabilistic description of polarization is unavoidable in quantum optics from the very beginning. This is because the Stokes operators do not commute and thus no state can have a definite value of all them simultaneously (except the two-mode vacuum). No state has a definite polarization ellipse for the same reasons that quantum particles do not follow definite classical trajectories. A suitable correspondence between light states and polarization distributions is discussed in Sec. II.

Among other applications, this formalism allows us to introduce a suitable definition of the degree of polarization that avoids the difficulties that the classical definition encounters. The degree of polarization can be defined as the distance between the polarization distribution and the uniform distribution corresponding to unpolarized light. In this way, the degree of polarization depends on all moments of the Stokes operators, and not only on the first one. This definition is presented and their properties examined in Sec. III. In Sec. IV, we apply this formalism to some interesting quan-

*Electronic address: alluis@ fis.ucm.es tum states of light such as $\mathrm{SU}(2)$ coherent, squeezed, number, and phase states.

\section{PROBABILITY DISTRIBUTION FOR POLARIZATION}

In this section, we develop the main definitions required for later sections. We assume a monochromatic plane wave propagating in the $z$ direction whose electric field lies in the $x y$ plane. In these conditions, we are dealing with a twomode field that can be fully described by two complex amplitude operators. They are denoted by $a_{+}, a_{-}$when using the basis of circular polarizations while they are denoted by $a_{x}, a_{y}$ when using the basis of linear polarization along the $x$ and $y$ axes, respectively, so that

$$
a_{ \pm}=\frac{1}{\sqrt{2}}\left(a_{x} \pm i a_{y}\right)
$$

The Stokes operators are defined as the quantum counterparts of the classical variables as

$$
\begin{gathered}
S_{0}=a_{+}^{\dagger} a_{+}+a_{-}^{\dagger} a_{-}, \quad S_{x}=a_{+}^{\dagger} a_{-}+a_{-}^{\dagger} a_{+}, \\
S_{y}=i\left(a_{-}^{\dagger} a_{+}-a_{+}^{\dagger} a_{-}\right), \quad S_{z}=a_{+}^{\dagger} a_{+}-a_{-}^{\dagger} a_{-},
\end{gathered}
$$

and their mean values are the Stokes parameters $\left\langle S_{0}\right\rangle,\langle\boldsymbol{S}\rangle$. They satisfy the commutation relations of an angular momentum,

$$
\left[S_{x}, S_{y}\right]=2 i S_{z}, \quad\left[S, S_{0}\right]=\mathbf{0} .
$$

Among other consequences, this implies that no field state (leaving aside the two-mode vacuum) can have definite nonfluctuating values of all the Stokes operators simultaneously. This is expressed by the uncertainty relation [11]

$$
(\Delta S)^{2}=\left(\Delta S_{x}\right)^{2}+\left(\Delta S_{y}\right)^{2}+\left(\Delta S_{z}\right)^{2} \geqslant 2\left\langle S_{0}\right\rangle,
$$

where $(\Delta A)^{2}=\left\langle A^{2}\right\rangle-\langle A\rangle^{2}$. We stress that this applies to a two-mode field. For multimode fields, things can be slightly different [5]. In sharp contrast to classical optics, the electric vector of a monochromatic field never describes a definite ellipse [12]. As a matter of fact, the probability distribution for the electric field can be very far from having an elliptical form, as we will show in Sec. IV. 
All this means that the polarization must be unavoidably described in terms of a probability distribution of polarization states, i.e., a probability distribution on the surface of the Poincare sphere. Since there is no sharp correspondence between quantum states and polarization states, there cannot be a straightforward definition of such a distribution. The identification of the Stokes operators as an angular momentum allows us to benefit from the solutions proposed for the representation of spin systems by quasidistributions on the sphere. Different correspondences have been proposed [13$15]$ including discrete versions [16-19]. Maybe the best behaved for our purposes is the $\mathrm{SU}(2) Q$ function defined as [15]

$$
Q(\theta, \phi)=\sum_{n=0}^{\infty} \frac{n+1}{4 \pi}\langle n, \theta, \phi|\rho| n, \theta, \phi\rangle,
$$

where $\rho$ is the density matrix for the two-mode field, $|n, \theta, \phi\rangle$ are the $\mathrm{SU}(2)$ coherent states,

$$
\begin{aligned}
|n, \theta, \phi\rangle= & \sum_{m=0}^{n}\left(\begin{array}{l}
n \\
m
\end{array}\right)^{1 / 2}\left(\sin \frac{\theta}{2}\right)^{n-m}\left(\cos \frac{\theta}{2}\right)^{m} e^{-i m \phi}|m\rangle_{+} \mid n \\
& -m\rangle_{-},
\end{aligned}
$$

and $|m\rangle_{+}|n-m\rangle_{-}$denote photon number states in the corresponding mode. In these expressions, $\theta$ and $\phi$ are the polar and the azimuthal angles, respectively, of the Poincare sphere. The SU(2) coherent states are eigenstates of the total number operator $S_{0}|n, \theta, \phi\rangle=n|n, \theta, \phi\rangle$. Therefore, the $n$ sum in Eq. (5) removes the total intensity of the field so that $Q(\theta, \phi)$ contains only the polarization properties.

It is worth noting that the $\mathrm{SU}(2)$ coherent states are the only states reaching the equality in the uncertainty relation (4) [11]. Therefore, the $Q$ function is the projection on the states having the most definite polarization state allowed by the quantum theory. We will see in the next section that other approaches confirm the minimum polarization fluctuations of the $\mathrm{SU}(2)$ coherent states.

The SU(2) $Q$ function defined in Eq. (5) has a direct relationship with the more standard $\mathcal{Q}$ function $\mathcal{Q}(\alpha, \beta)$ $=\langle\alpha, \beta|\rho| \alpha, \beta\rangle$ defined in terms of the quadrature coherent states $|\alpha, \beta\rangle=|\alpha\rangle_{+}|\beta\rangle_{-}$with

$$
|\alpha\rangle_{+}=e^{-|\alpha|^{2} / 2} \sum_{n=0}^{\infty} \frac{\alpha^{n}}{\sqrt{n !}}|n\rangle_{+},
$$

and similarly for $|\beta\rangle_{-}$[20]. The product of quadrature coherent states can be expressed as a Poissonian superposition of SU(2) coherent states [21],

$$
|\alpha, \beta\rangle=e^{-r^{2} / 2} \sum_{n=0}^{\infty} \frac{r^{n} e^{i n \delta}}{\sqrt{n !}}|n, \theta, \phi\rangle,
$$

where the state parameters are connected by the relations

$$
\alpha=r \sin \frac{\theta}{2} e^{i \delta} e^{-i \phi}, \quad \beta=r \cos \frac{\theta}{2} e^{i \delta}
$$

so that

$$
Q(\theta, \phi)=\frac{1}{4} \int_{2 \pi} d \delta \int_{0}^{\infty} d r r^{3} \mathcal{Q}(\alpha, \beta) .
$$

The $Q$ function serves also to find the polarization states that are closest to a given field state. This can be achieved by finding the $\theta, \phi$ values for which $Q$ is maximum. In general, the solution is not unique, especially if $\langle\boldsymbol{S}\rangle=\mathbf{0}$.

As we have mentioned above, the $\mathrm{SU}(2) Q$ function is not the unique correspondence between spin states and functions on the sphere. However, we think that this is the best choice for our purposes. Other options (such as the diagonal representation in the coherent state basis or the Wigner function) can be very singular and far from classical intuition, they can take negative values, and they can be void of practical measurement. Plots of the SU(2) Wigner function for some quantum states can be found in Ref. [22].

In addition to the Poincare sphere, it is also customary to picture the polarization state in terms of the trajectory described by the electric field. In quantum terms, there are no trajectories and we must deal with probability distributions. The electric field in the $x y$ plane is represented by the adimensional quadrature operators

$$
\hat{x}=\frac{1}{2}\left(a_{x}+a_{x}^{\dagger}\right), \quad \hat{y}=\frac{1}{2}\left(a_{y}+a_{y}^{\dagger}\right),
$$

which are the real parts of the corresponding complex amplitude operators. The probability distribution $\mathcal{P}(x, y)$ is given by

$$
\mathcal{P}(x, y)=\langle x, y|\rho| x, y\rangle,
$$

where $x, y$ and $|x, y\rangle$ are the eigenvalues and eigenvectors, respectively, of $\hat{x}, \hat{y}$. In general, this probability distribution varies with time. However, in this work we will consider only stationary states of the free evolution (i.e., eigenstates of the total number operator $\left.S_{0}\right)$ so that $\mathcal{P}(x, y)$ will not depend on time.

\section{DEGREE OF POLARIZATION}

The classical definition of the degree of polarization is

$$
P_{\text {class }}=\frac{\sqrt{\langle\boldsymbol{S}\rangle^{2}}}{\left\langle S_{0}\right\rangle} .
$$

We have already discussed that this definition is not fully satisfactory since $P_{\text {class }}$ is defined solely in terms of the first moment of the Stokes operators and this cannot reflect polarization properties defined in terms of higher-order moments. In particular, there are states with $P_{\text {class }}=0$ that cannot be regarded as being unpolarized. Moreover, the definition (13) does not reflect the lack of perfect polarization of every quantum state. For example, the $\mathrm{SU}(2)$ coherent states reach perfect classical polarization $P_{\text {class }}=1$ and this includes the two-mode vacuum [23]. A definition close to Eq. (13) that does not present this last problem has been used in Ref. [19]. 
Next we introduce a definition of the degree of polarization that avoids these difficulties. The degree of polarization of a given field state can be naturally defined as the distance between its $Q$ function and the $Q$ function for unpolarized light. The unpolarized light is defined as the field states with a uniform distribution,

$$
Q_{\text {unpol }}(\theta, \phi)=\frac{1}{4 \pi}
$$

that fully agrees with more involved approaches [2].

We define the distance

$$
\begin{aligned}
D & =4 \pi \int d \Omega\left[Q(\theta, \phi)-\frac{1}{4 \pi}\right]^{2} \\
& =4 \pi \int d \Omega[Q(\theta, \phi)]^{2}-1,
\end{aligned}
$$

where $d \Omega=\sin \theta d \theta d \phi$ is the differential of solid angle. It can be seen that $D$ ranges from 0 to $\infty$. We normalize it defining the degree of polarization as

$$
P=\frac{D}{1+D}=1-\frac{1}{4 \pi} \Sigma,
$$

where

$$
\Sigma=\frac{1}{\int d \Omega[Q(\theta, \phi)]^{2}},
$$

so that $1 \geqslant P \geqslant 0$.

Next we analyze the main properties of this definition. First we note that the only states with $P=0$ are the unpolarized states with $Q=1 /(4 \pi)$. In contrast to the classical definition, we will see in the next section that there are field states with $\langle\boldsymbol{S}\rangle=\mathbf{0}$ and $P \neq 0$. This occurs because $P$ is a function of all moments of the Stokes operators and not only of the first one.

The definitions (15) and (16) are invariant under SU(2) transformations applied to the field state. This means that the degree of polarization depends on the form of the $Q$ function, but not on its position or orientation on the Poincare sphere. In practical terms, the $\mathrm{SU}(2)$ transformations are linear and energy-conserving transformations of the complex amplitude operators. They are produced by passive optical devices such as the free propagation, beam splitters, phase plates, and mirrors [24].

The function $\Sigma$ in Eq. (17) can be interpreted as the effective area where the $Q$ function is different from zero. In other words, $\Sigma$ is a measure of the number of polarization states contained in a given field state. For example, if $Q$ $=1 / \sigma$ on a surface of area $\sigma$ and $Q=0$ outside it, then $\Sigma$ $=\sigma$.

This and similar definitions have already been used as measures of localization and uncertainty in different contexts
[17,25-28]. In particular, Eq. (15) can be regarded as a particular case of a general class of measures of localization $[26,28]$,

$$
M_{r}=\left(\int d \Omega Q^{1+r}\right)^{1 / r}
$$

that includes the Wehrl and Shannon entropies [29],

$$
\lim _{r \rightarrow 0} \ln M_{r}=\int d \Omega Q \ln Q
$$

where $Q$ represents here a general probability distribution. In our case, we have $D=4 \pi M_{1}-1$ and $\Sigma=1 / M_{1}$. This identification endows our definitions with desirable properties such as the ones listed in Ref. [26].

It is interesting to ask for the states with maximum $P$. In the next section, we show that there are different states that satisfy $P \rightarrow 1$ when their intensity is arbitrarily increased. On the other hand, the states with maximum degree of polarization when the intensity is kept fixed are the SU(2) coherent states. This is because it has been recently shown that these are the most localized quantum states for spin systems [28] (Lieb's conjecture [30,31]). This is consistent with the definition of the polarization distribution by projection on the SU(2) coherent states.

We should mention that the Wigner function has already been used as a measure of the area occupied by quantum states [25]. However, it must be noticed that for the $\mathrm{SU}(2)$ Wigner function $W(\theta, \phi)[13]$ we have that $\int d \Omega[W(\theta, \phi)]^{2}$ takes exactly the same value for all pure states so that this provides a measure of purity of quantum states rather than a measure of polarization.

We conclude this section discussing the feasibility of the experimental determination of $P$. Among the diverse theoretical proposals that may serve to estimate $P$, we restrict ourselves to methods already implemented in practice. In this sense, perhaps the most direct relation of $P$ with measurable quantities is given by Eq. (10) relating the $\mathrm{SU}(2) Q$ function to the quadrature $Q$ function $\mathcal{Q}$. We can mention two strategies for the experimental determination of $\mathcal{Q}$.

The probability distribution $\mathcal{Q}$ can be measured by using a double homodyne detector for each field mode [32]. This is a conceptually simple scheme that provides a direct measurement of $\mathcal{Q}(\alpha, \beta)$ at each point $(\alpha, \beta)$ without involving further data analysis. The practical feasibility of double homodyne detection has been demonstrated in Refs. [33,34]. On the other hand, $\mathcal{Q}(\alpha, \beta)$ can also be determined by using tomographic reconstruction methods based on single homodyne detection [35]. This has been carried out experimentally for single mode fields in Refs. [36,37]. It is worth noting that both schemes are equally valid for classical as well as for quantum fields since they rely on the measurement of the complex amplitudes of the field modes. The classical and quantum regimes only differ in the properties of the output statistics.

From a practical perspective, the determination of $\mathcal{Q}$ and $P$ will be affected by experimental errors such as statistical fluctuations, inefficient detection, finite sampling, and ther- 
mal noise. Since a detailed examination of these errors is beyond the scope of this work, we just provide some comments in support of the viability of the measurement of $P$.

Homodyne detection (single and double) is a wellestablished technique. The precision of current experimental arrangements allows the accurate determination of rather involved quantities such as quantum phase distributions and number-phase uncertainty relations as demonstrated in Refs. $[34,37]$. In our case, the relation between the desired quantity $P$ and the statistics of the measurement is no more complex than in the examples just quoted. Therefore, it should be possible to estimate $P$ accurately using current technology.

The effect of experimental uncertainties in homodyne detection and their compensation have been well studied [38]. From these analyses it appears that tomographic methods based on single homodyne detection are superior to the direct double homodyne measurement of $\mathcal{Q}$. This is because $\mathcal{Q}$ is an intrinsically smooth function that requires large data sampling in order to obtain relevant information about the input state. This hinders the correction and compensation of experimental errors.

Finally, we can note that we are not interested in all the information carried by $\mathcal{Q}$, but only in the integration in Eq. (15). This implies that the statistical fluctuations may cancel out partially when performing the effective averaging process involved in the definition of $P$.

\section{APPLICATIONS}

In this section, we apply the ideas of the preceding sections to some relevant field states. Throughout we take advantage of the SU(2) invariance to choose the simplest expressions. It is worth pointing out that all the probability distributions examined below are time-independent under free evolution because the corresponding states are eigenstates of the total number operator $S_{0}$. This also means that all fluctuations and fuzziness in the following examples are due solely to polarization fluctuations and cannot be ascribed to intensity fluctuations.

\section{A. SU(2) coherent states}

The $Q$ function for the $\mathrm{SU}(2)$ coherent state $\mid n, \theta=0$, $\phi\rangle=|n\rangle_{+}|0\rangle_{-}$is

$$
Q(\theta, \phi)=\frac{n+1}{4 \pi}\left(\cos \frac{\theta}{2}\right)^{2 n} .
$$

The degree of polarization and the effective area of the Poincaré sphere occupied by these states are [28,31]

$$
P=\left(\frac{n}{n+1}\right)^{2}, \quad \Sigma=4 \pi \frac{2 n+1}{(n+1)^{2}},
$$

while $P_{\text {class }}=1$ for all $n$. The particular case $n=0$ is the two-mode vacuum with $P=0$ and $\Sigma=4 \pi$, as could be expected. On the other hand, when $n \rightarrow \infty$ we have $P \rightarrow 1$ and

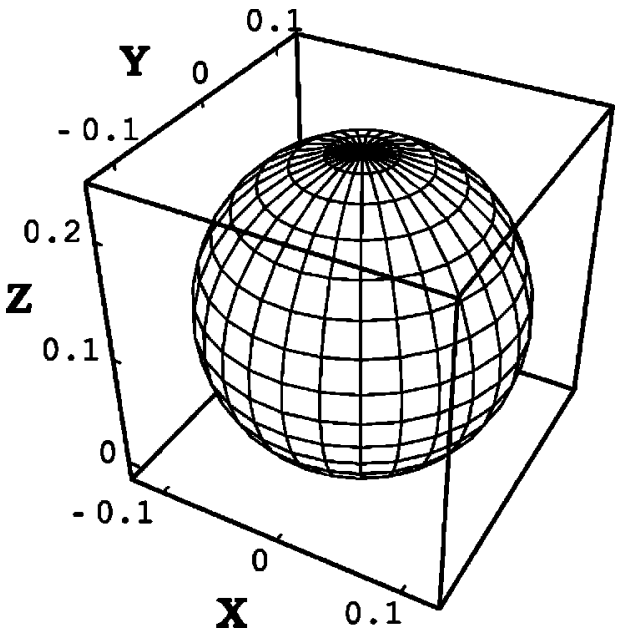

FIG. 1. Spherical plot of the $Q$ function for a SU(2) coherent state with $n=2$ and $\theta=0$. Note that the origin is at the bottom of the figure. It can be seen that it does not depend on $\phi$ and that it is located around the north pole of the Poincare sphere.

$\Sigma \rightarrow 0$. In the limit of high intensity, the $\mathrm{SU}(2)$ coherent states tend to be fully polarized and their $Q$ function tends to be a $\delta$ function.

In Fig. 1, we have represented $Q(\theta, \phi)$ for the particular case $|2\rangle_{+}|0\rangle_{-}$. It can be seen that it is localized around the north pole of the Poincare sphere that corresponds to circular polarization (note that the origin is at the bottom of the figure). Nevertheless, the fluctuations around this point are large enough to decrease the degree of polarization to $P$ $=\frac{4}{9}$. In Fig. 2, we have plotted the probability distribution for the field quadratures $\mathcal{P}(x, y)$ in the state $|2\rangle_{+}|0\rangle_{-}$,

$$
\mathcal{P}(x, y)=\frac{4}{\pi}\left(x^{2}+y^{2}\right)^{2} e^{-2\left(x^{2}+y^{2}\right)},
$$

where it can be seen that it resembles a circular trajectory. Similar plots can be found in Ref. [12].

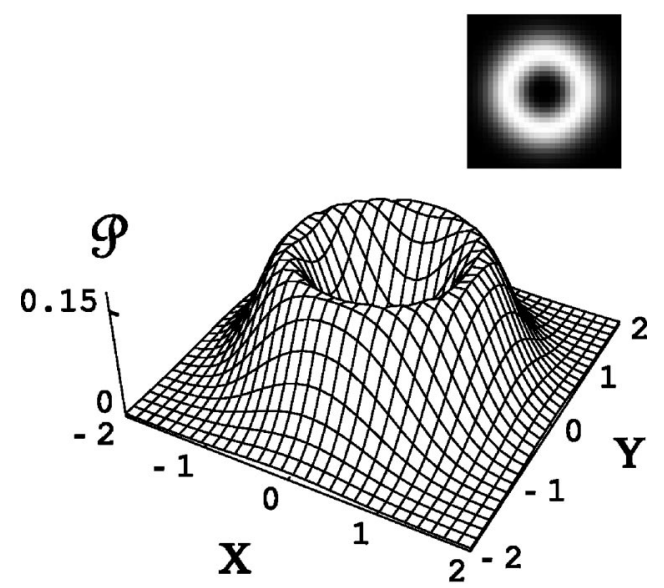

FIG. 2. Probability distribution for the field quadratures $x, y$ for a $\mathrm{SU}(2)$ coherent state with $n=2$ and $\theta=0$. The inset shows a density plot. It can be seen that this is close to a circularly polarized state. 


\section{B. SU(2) squeezed states}

Several definitions of polarization or spin squeezing can be found in the literature $[19,39]$. Here we focus on the one relevant in the context of precision phase-shift measurements and atomic spectroscopy. The so-called spectroscopic squeezing occurs when, for fixed $n$, the uncertainty $\Delta S_{\perp}$ of a component of the Stokes operators normal to $\langle\boldsymbol{S}\rangle$ satisfies that [7-9]

$$
\frac{\Delta S_{\perp}}{|\langle S\rangle|}<\frac{1}{\sqrt{n}}
$$

The equality is reached by the SU(2) coherent states. The states that satisfy the inequality are the solutions of the eigenvalue equation

$$
\left(\lambda S_{+}+\frac{1}{\lambda} S_{-}\right)|\xi\rangle=m|\xi\rangle
$$

where $S_{ \pm}=S_{x} \pm i S_{y}$ and $\lambda, m$ are constants. The solution of this equation can be found in Ref. [7], where it is shown that maximum squeezing occurs when $\lambda \rightarrow 1$ and $m \rightarrow 0$. For simplicity, here we consider the case $n=2$ and $m=0$. In such a case the solution of the eigenvalue equation in the photonnumber basis is

$$
|\xi\rangle=\sin \xi|2\rangle_{+}|0\rangle_{-}-\cos \xi|0\rangle_{+}|2\rangle_{-},
$$

where $\tan \xi=\lambda^{2}$. The degree of polarization is

$$
P=1-\frac{10}{3} \frac{1}{5+\cos (4 \xi)} .
$$

For these states, $\left\langle S_{x}\right\rangle=\left\langle S_{y}\right\rangle=0$. If we take $S_{\perp}=S_{x}$, the condition (23) becomes

$$
\frac{\Delta S_{x}}{\left|\left\langle S_{z}\right\rangle\right|}=\frac{1}{\sqrt{2}|\sin \xi+\cos \xi|}<\frac{1}{\sqrt{2}}
$$

which is satisfied when $\pi / 2>\xi>0$. When $\xi=0, \pi / 2$, the states (25) are SU(2) coherent states with the maximum degree of polarization $\left(P=\frac{4}{9}\right)$. On the other hand, maximum squeezing occurs for $\xi=\pi / 4$, leading to a minimum degree of polarization $P=\frac{1}{6}$.

The state with maximum squeezing is

$$
|\xi\rangle=\frac{1}{\sqrt{2}}\left(|2\rangle_{+}|0\rangle_{-}-|0\rangle_{+}|2\rangle_{-}\right)=|1\rangle_{x}|1\rangle_{y},
$$

where $|1\rangle_{x}|1\rangle_{y}$ are photon number states in modes $a_{x}, a_{y}$. This is the eigenstate of $S_{x}$ with eigenvalue zero. Its quantum polarization properties have been studied in Refs. [3,4]. The $\mathrm{SU}(2) Q$ function for the state (28),

$$
Q(\theta, \phi)=\frac{3}{8 \pi}\left(\cos ^{2} \theta \cos ^{2} \phi+\sin ^{2} \phi\right),
$$

is represented in Fig. 3, where it can be appreciated that the

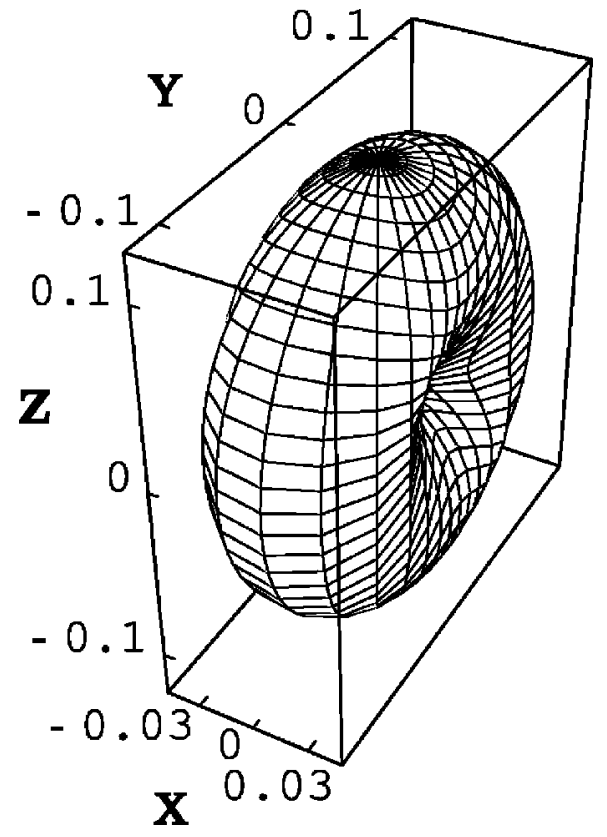

FIG. 3. Spherical plot of the $Q$ function for a SU(2) squeezed state with $n=2, \lambda=1$, and $m=0$. Compared to the coherent state, it can be seen that the fluctuations of $S_{x}$ are clearly reduced.

fluctuations of $S_{x}$ are reduced in comparison with the coherent state in Fig. 1. The probability distribution for the field quadratures,

$$
\mathcal{P}(x, y)=\frac{32}{\pi} x^{2} y^{2} e^{-2\left(x^{2}+y^{2}\right)},
$$

is represented in Fig. 4, where it can be seen that it does not resemble an ellipse. It is worth noting that for the state (28), the Stokes parameters vanish, $\langle\boldsymbol{S}\rangle=\mathbf{0}$, so it would be unpolarized according to the classical definition.

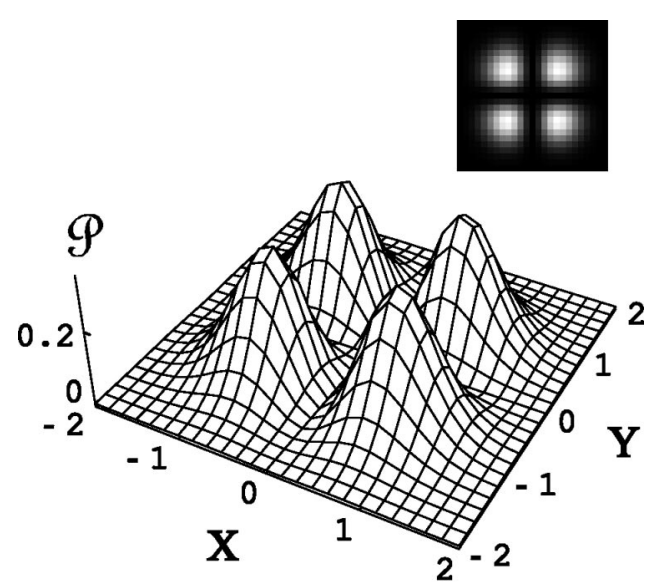

FIG. 4. Probability distribution for the field quadratures $x, y$ for a $\mathrm{SU}(2)$ squeezed state with $n=2, \lambda=1$, and $m=0$. The inset shows a density plot. This distribution does not resemble any classical polarization ellipse. 


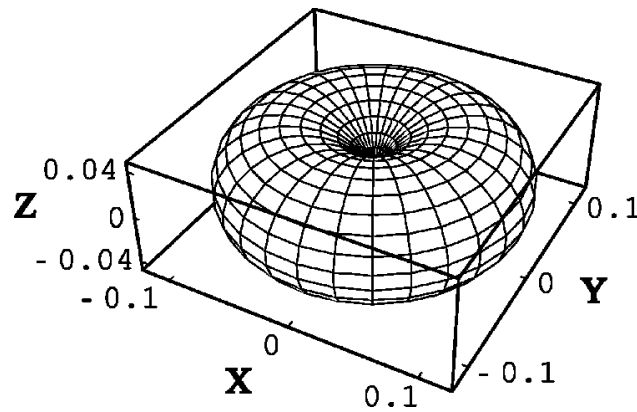

FIG. 5. Spherical plot of the $Q$ function for a number state $|1\rangle_{+}|1\rangle_{-}$. It can be seen that it does not depend on $\phi$ and that it is located around the equator.

\section{Number states}

The $Q$ function for the number state $|m\rangle_{+}|n-m\rangle_{-}$is

$$
Q(\theta, \phi)=\frac{n+1}{4 \pi}\left(\begin{array}{l}
n \\
m
\end{array}\right)\left(\sin \frac{\theta}{2}\right)^{2(n-m)}\left(\begin{array}{r}
\cos \frac{\theta}{2} \\
)^{2 m},
\end{array}\right.
$$

and the degree of polarization is $[28,31]$

$$
P=1-\frac{2 n+1}{(n+1)^{2}}\left(\begin{array}{c}
2 n \\
2 m
\end{array}\right)\left(\begin{array}{l}
n \\
m
\end{array}\right)^{-2} .
$$

We can briefly examine the limit of large total photon number. If for simplicity we take $n=2 m$, the Stirling approximation when $n \gg 1$ leads to

$$
P \simeq 1-\sqrt{\frac{\pi}{n}}
$$

Therefore, the number states also tend to be fully polarized $(P \rightarrow 1)$ when their intensity is increased. We note that for the states $|m\rangle_{+}|m\rangle_{-}$we have $\langle\boldsymbol{S}\rangle=\mathbf{0}$, so classically they would be unpolarized for every $m$, even in the limit $m \gg 1$.

For $n=2$ and $m=1$, we have

$$
|\psi\rangle=|1\rangle_{+}|1\rangle_{-}=\frac{1}{\sqrt{2}}\left(|2\rangle_{x}|0\rangle_{y}+|0\rangle_{x}|2\rangle_{y}\right) .
$$

Its $Q$ function

$$
Q(\theta, \phi)=\frac{3}{8 \pi}(\sin \theta)^{2}
$$

is represented in Fig. 5, where it can be seen that it is a rotated version of Fig. 3 corresponding to the squeezed state (28). Accordingly, the degree of polarization is again $P=\frac{1}{6}$. The $Q$ function is located around the equator $\theta=\pi / 2$ so this state can be regarded as an equally weighted superposition of all linearly polarized states. The probability distribution for the field quadratures plotted in Fig. 6,

$$
\mathcal{P}(x, y)=\frac{2}{\pi}\left[2\left(x^{2}+y^{2}\right)-1\right]^{2} e^{-2\left(x^{2}+y^{2}\right)},
$$

resembles circular polarization [6].

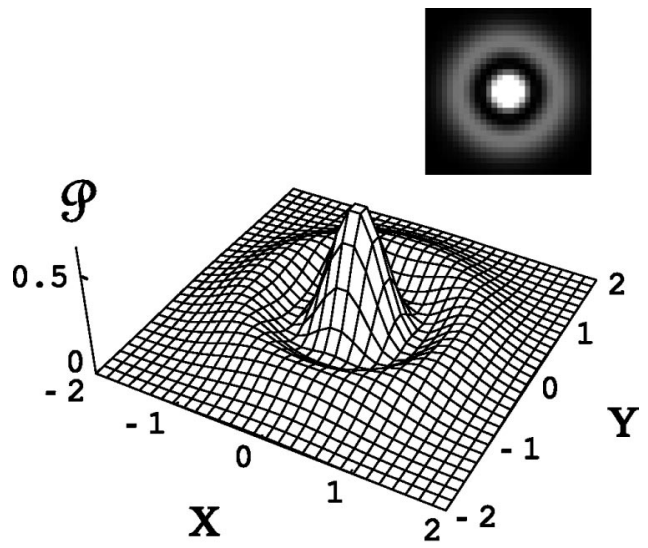

FIG. 6. Probability distribution for the field quadratures $x, y$ for the number state $|1\rangle_{+}|1\rangle_{-}$. The inset shows a density plot. This distribution resembles circular polarization.

\section{Phase states}

The variable complementary to number is the phase. In the context of polarization, the relevant phase variable is the phase difference, which corresponds to the azimuthal angle $\phi$ on the Poincare sphere. There are several approaches to the quantum description of the phase difference in quantum optics [9]. Most of them conclude that the phase-difference states are

$$
|n, \varphi\rangle=\frac{1}{\sqrt{n+1}} \sum_{m=0}^{n} e^{-i m \varphi}|m\rangle_{+}|n-m\rangle_{-} .
$$

The $Q$ function for $n=2$ and $\varphi=0$,

$$
Q(\theta, \phi)=\frac{1}{4 \pi}\left[\left(\cos \phi+\frac{1}{\sqrt{2}} \sin \theta\right)^{2}+\sin ^{2} \phi \cos ^{2} \theta\right],
$$

is plotted in Fig. 7, where it can be seen that it is centered

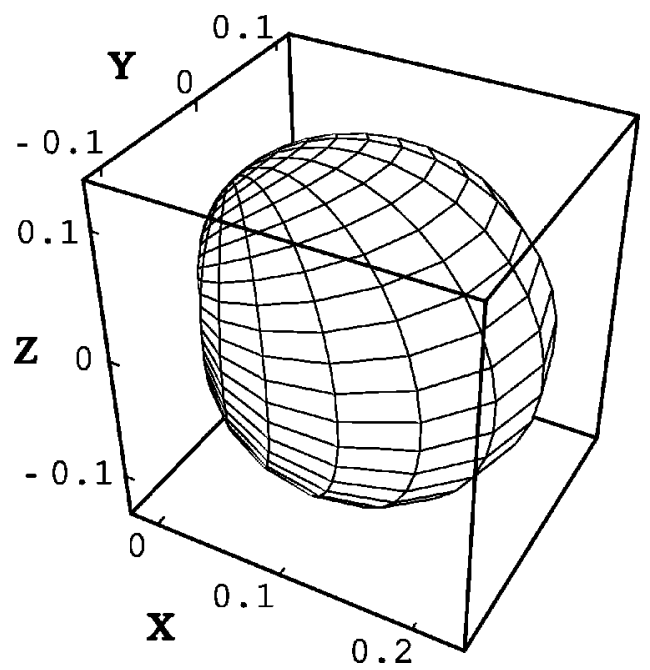

FIG. 7. Spherical plot of the $Q$ function for the phase state $\mid n$ $=2, \varphi=0\rangle$. Note that the origin is situated at the left of the figure. It can be appreciated that it is centered around $\phi=0$. 
around $\phi=0$ (note that the origin is at the left of the figure). The degree of polarization is $P=\frac{11}{26}$, which is close to the maximum for $n=2\left(P=\frac{4}{9}\right)$. The probability distribution for the field quadratures,

$$
\begin{aligned}
\mathcal{P}(x, y)= & \frac{2}{3 \pi}\left[2(1+\sqrt{2}) x^{2}\right. \\
& \left.+2(1-\sqrt{2}) y^{2}-1\right]^{2} e^{-2\left(x^{2}+y^{2}\right)},
\end{aligned}
$$

is plotted in Fig. 8.

\section{CONCLUSIONS}

Quantum optics entails polarization states that cannot be suitably described by the classical formalism based on the Stokes parameters. In this work, we have analyzed the polarization of quantum states in terms of a suitably defined probability distribution on the Poincare sphere. This allows us to define the degree of polarization as the distance to the uniform distribution representing unpolarized light. With this natural definition, the degree of polarization turns out to de-
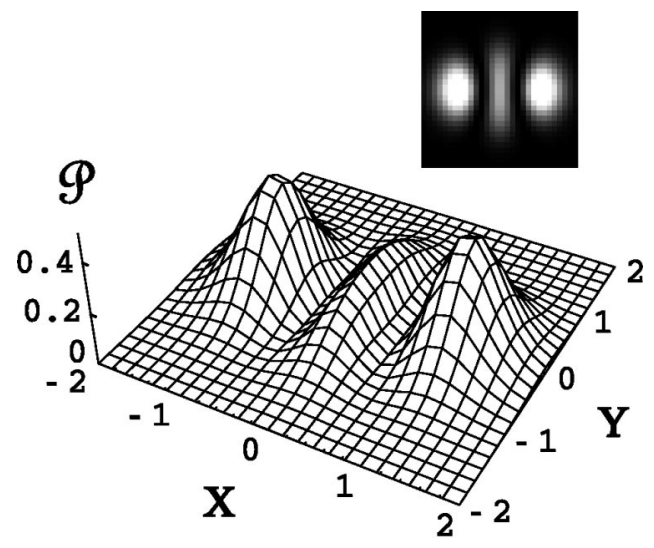

FIG. 8. Probability distribution for the field quadratures $x, y$ for the phase state $|n=2, \varphi=0\rangle$. The inset shows a density plot.

pend on the area of the Poincare sphere occupied by the field state, i.e., the number of classical polarization states that it contains. We have shown that these definitions are well behaved even when the classical formalism fails.
[1] M. Born and E. Wolf, Principles of Optics (Pergamon, Oxford, 1980); L. Mandel and E. Wolf, Optical Coherence and Quantum Optics (Cambridge University Press, Cambridge, England, 1995).

[2] H. Prakash and N. Chandra, Phys. Rev. A 4, 796 (1971); G.S. Agarwal, Lett. Nuovo Cimento Soc. Ital. Fis. 1, 53 (1971); J. Söderholm, G. Björk, and A. Trifonov, Opt. Spectrosc. 91, 532 (2001); e-print quant-ph/0007099; J. Lehner, U. Leonhardt, and H. Paul, Phys. Rev. A 53, 2727 (1996).

[3] D.N. Klyshko, Phys. Lett. A 163, 349 (1992).

[4] P. Usachev, J. Söderholm, G. Björk, and A. Trifonov, Opt. Commun. 193, 161 (2001).

[5] V.P. Karassiov, J. Phys. A 26, 4345 (1993); V.P. Karasev and A.V. Masalov, Opt. Spectrosc. 74, 551 (1993).

[6] T. Tsegaye, J. Söderholm, M. Atatüre, A. Trifonov, G. Björk, A.V. Sergienko, B.E.A. Saleh, and M.C. Teich, Phys. Rev. Lett. 85, 5013 (2000).

[7] M. Hillery and L. Mlodinow, Phys. Rev. A 48, 1548 (1993); G.S. Agarwal and R.R. Puri, ibid. 49, 4968 (1994); C. Brif and A. Mann, ibid. 54, 4505 (1996).

[8] M. Kitagawa and M. Ueda, Phys. Rev. A 47, 5138 (1993); D.J. Wineland, J.J. Bollinger, W.M. Itano, and D.J. Heinzen, ibid. 50, 67 (1994).

[9] A. Luis and L.L. Sánchez-Soto, Prog. Opt. 41, 421 (2000).

[10] P. Grangier, R.E. Slusher, B. Yurke, and A. LaPorta, Phys. Rev. Lett. 59, 2153 (1987); W.P. Bowen, R. Schnabel, H.-A. Bachor, and P.K. Lam, e-print quant-ph/0110129.

[11] R. Delbourgo, J. Phys. A 10, 1837 (1977).

[12] J. Pollet, O. Méplan, and C. Gignoux, J. Phys. A 28, 7287 (1995).

[13] G.S. Agarwal, Phys. Rev. A 24, 2889 (1981); J.C. Várilly and J.M. Gracia-Bondía, Ann. Phys. (N.Y.) 190, 107 (1989).

[14] G. Ramachandran, A.R. Usha Devi, P. Devi, and S. Sirsi,
Found. Phys. 26, 401 (1996); V.V. Dodonov and V.I. Man'ko, Phys. Lett. A 229, 335 (1997).

[15] F.T. Arecchi, E. Courtens, R. Gilmore, and H. Thomas, Phys. Rev. A 6, 2211 (1972).

[16] W.K. Wootters, Ann. Phys. (N.Y.) 176, 1 (1987); O. Cohendet, Ph. Combe, M. Sirugue, and M. Sirugue-Collin, J. Phys. A 21, 2875 (1988); D. Galetti and A.F.R. Toledo Piza, Physica A A149, 267 (1988); J.A. Vaccaro and D.T. Pegg, Phys. Rev. A 41, 5156 (1990); P. Kasperkovitz and M. Peev, Ann. Phys. (N.Y.) 230, 21 (1994); U. Leonhardt, Phys. Rev. Lett. 74, 4101 (1995); Phys. Rev. A 53, 2998 (1996); A. Luis and J. Peřina, J. Phys. A 31, 1423 (1998).

[17] A. Lukš and V. Peřinová, Quantum Opt. 6, 125 (1994).

[18] T. Hakiog̃lu, A.S. Shumovsky, and O. Aytür, Phys. Lett. A 194, 304 (1994); A. Luis and J. Perina, Quantum Semiclassic. Opt. 8, 887 (1996); T. Hakioğlu, Phys. Rev. A 59, 1586 (1999).

[19] A.P. Alodjants and S.M. Arakelian, J. Mod. Opt. 46, 475 (1999).

[20] J. Peřina, Quantum Statistics of Linear and Nonlinear Optical Phenomena (Kluwer, Dordrecht, 1991).

[21] P.W. Atkins and J.C. Dobson, Proc. R. Soc. London, Ser. A 321, 321 (1971).

[22] J.P. Dowling, G.S. Agarwal, and W.P. Schleich, Phys. Rev. A 49, 4101 (1994).

[23] R. Tanaś and S. Kielich, J. Mod. Opt. 37, 1935 (1990).

[24] B. Yurke, S.L. McCall, and J.R. Klauder, Phys. Rev. A 33, 4033 (1986); R.A. Campos, B.E.A. Saleh, and M.C. Teich, ibid. 40, 1371 (1989); U. Leonhardt, ibid. 48, 3265 (1993); A. Luis and L.L. Sánchez-Soto, Quantum Semiclassic. Opt. 7, 153 (1995).

[25] E.J. Heller, Phys. Rev. A 35, 1360 (1987).

[26] H. Maassen and J.B.M. Uffink, Phys. Rev. Lett. 60, 1103 (1988).

[27] I. Bialynicki-Birula, M. Freyberger, and W. Schelich, Phys. 
Scr., T48, 113 (1993); B. Mirbach and H.J. Korsch, Ann. Phys. (N.Y.) 265, 80 (1998); A. Anderson and J.J. Halliwell, Phys. Rev. D 48, 2753 (1993); A. Sugita and H. Aiba, e-print nlin.CD/0106012; C. Brukner and A. Zeilinger, Phys. Rev. Lett. 83, 3354 (1999); M.J.W. Hall, Phys. Rev. A 59, 2602 (1999).

[28] S. Gnutzmann and K. Życzkowski, J. Phys. A 34, 10123 (2001).

[29] A. Wehrl, Rev. Mod. Phys. 50, 221 (1978); Rep. Math. Phys. 30, 119 (1991).

[30] E.H. Lieb, Commun. Math. Phys. 62, 35 (1978).

[31] H. Scutaru, e-print math-ph/9909024.

[32] N.G. Walker, J. Mod. Opt. 34, 15 (1987); Y. Lai and H.A. Haus, Quantum Opt. 1, 99 (1989); U. Leonhardt and H. Paul, Phys. Rev. A 47, R2460 (1993); J. Mod. Opt. 40, 1745 (1993); M. Freyberger and W. Schleich, Phys. Rev. A 47, R30 (1993); M. Freyberger, K. Vogel, and W.P. Schleich, Quantum Opt. 5, 65 (1993); Phys. Lett. A 176, 41 (1993).

[33] N.G. Walker and J.E. Carroll, Electron. Lett. 20, 981 (1984); Opt. Quantum Electron. 18, 355 (1986).

[34] J.R. Torgerson and L. Mandel, Phys. Rev. Lett. 76, 3939 (1996).

[35] K. Vogel and H. Risken, Phys. Rev. A 40, 2847 (1989); U. Leonhardt, Measuring the Quantum State of Light (Cambridge University Press, Cambridge, England, 1997); D.-G. Welsch, W. Vogel, and T. Opatrný, in Progress in Optics, edited by E. Wolf (Elsevier Science, Amsterdam, 1999), Vol. 39; M.G. Raymer, D.F. McAlister, and U. Leonhardt, Phys. Rev. A 54, 2397 (1996); T. Opatrný, D.-G. Welsch, and W. Vogel, Opt. Commun. 134, 112 (1997).

[36] D.T. Smithey, M. Beck, M.G. Raymer, and A. Faridani, Phys.
Rev. Lett. 70, 1244 (1993); M. Munroe, D. Boggavarapu, M.E. Anderson, and M.G. Raymer, Phys. Rev. A 52, R924 (1995); S. Schiller, G. Breitenbach, S.F. Pereira, T. Müller, and J. Mlynek, Phys. Rev. Lett. 77, 2933 (1996); G. Breitenbach, S. Schiller, and J. Mlynek, Nature (London) 387, 471 (1997).

[37] M. Beck, D.T. Smithey, and M.G. Raymer, Phys. Rev. A 48, R890 (1993); D.T. Smithey, M. Beck, J. Cooper, and M.G. Raymer, ibid. 48, 3159 (1993); M. Beck, D.T. Smithey, J. Cooper, and M.G. Raymer, Opt. Lett. 18, 1259 (1993).

[38] U. Leonhardt and H. Paul, Phys. Rev. A 48, 4598 (1993); G.M. D'Ariano, C. Macchiavello, and M.G.A. Paris, Phys. Lett. A 195, 31 (1994); G.M. D'Ariano, U. Leonhardt, and H. Paul, Phys. Rev. A 52, R1801 (1995); T. Kiss, U. Herzog, and U. Leonhardt, ibid. 52, 2433 (1995); U. Herzog, ibid. 53, 1245 (1996); U. Leonhardt and M. Munroe, ibid. 54, 3682 (1996); P. Törmä, J. Mod. Opt. 43, 2437 (1996); S.M. Tan, ibid. 44, 2233 (1997); T. Opatrný and D.-G. Welsch, Phys. Rev. A 55, 1462 (1997); G.M. D’Ariano and M.G.A. Paris, Phys. Lett. A 233, 49 (1997); U. Leonhardt, M. Munroe, T. Kiss, Th. Richter, and M.G. Raymer, Opt. Commun. 127, 144 (1996); J. Fiurášek, M. Dakna, T. Opatrný, and D.-G. Welsch, Phys. Rev. A 62, 063811 (2000).

[39] D.F. Walls and P. Zoller, Phys. Rev. Lett. 47, 709 (1981); K. Wódkiewicz and J.H. Eberly, J. Opt. Soc. Am. B 2, 458 (1985); S.M. Barnett and M.-A. Dupertuis, ibid. 4, 505 (1987); M. Hillery, Phys. Rev. A 40, 3147 (1989); M.A. Dupertuis and A.N. Kireev, Quantum Opt. 2, 119 (1990); V.P. Karassiov, Phys. Lett. A 190, 387 (1994); N. Korolkova, G. Leuchs, R. Loudon, T.C. Ralph, and C. Silberhorn, e-print quant-ph/0108098. 